\title{
The role of lateral inhibition in the sensory processing in a simulated spiking neural controller for a robot
}

\author{
David Bowes, Rod Adams, Lola Cañamero, Volker Steuber, Neil Davey \\ Science and Technology Research Institute \\ University of Hertfordshire \\ College Lane \\ Hatfield,Hertfordshire \\ United Kingdom \\ Email: (D.H.Bowes, R.G.Adams, L.Cañamero, V.Steuber, N.Davey)@ herts.ac.uk
}

\begin{abstract}
Visual adaptation is the process that allows animals to be able to see over a wide range of light levels. This is achieved partially by lateral inhibition in the retina which compensates for low/high light levels. Neural controllers which cause robots to turn away from or towards light tend to work in a limited range of light conditions. In real environments, the light conditions can vary greatly reducing the effectiveness of the robot. Our solution for a simple Braitenberg vehicle is to add a single inhibitory neuron which laterally inhibits the output to the robot motors. This solution has additionally reduced the computational complexity of our simple neuron allowing for a greater number of neurons to be simulated with a fixed set of resources.
\end{abstract}

\section{INTRODUCTION}

$\mathbf{E}$ XPERIMENTS with robots (1) (2), in which interaction with a real environment takes place, usually involve many preliminary studies to determine the optimum calibration of the sensors for the extremes of the experimental conditions (3). In a previous experiment we investigated using different models of a simplified integrate and fire neuron in a Braitenberg vehicle. The experiment used excitatory, ipsilateral connections which resulted in negative phototaxis (4). We discovered that our model was not performing over the entire light intensity range due to neurons becoming saturated at high light levels and firing at their maximum rate. This could have been overcome by normalizing the input data which would have had to have been done prior to each experiment.

Real biological systems such as the retina are known to be able to adapt to different light conditions, allowing a person to be able to see in vastly different light conditions, with limited apparent impact on visual acuity. Such systems tend to include lateral inhibitory neurons that dampen output levels and enhance the contrast between neighboring areas of the visual field.

In this paper we investigate the effect of using neurons to laterally inhibit the input data, this allows our robot to orientate itself to darkness in a range of light conditions, thus improving on the results of previous experiments (5). To investigate the effect of lateral inhibition, these experiments were repeated with an extra neuron in a position similar to those found in the retina of an eye, see Fig. 1 and Fig. 2. 978-1-4244-2763-5/09/\$25.00 (C2009 IEEE

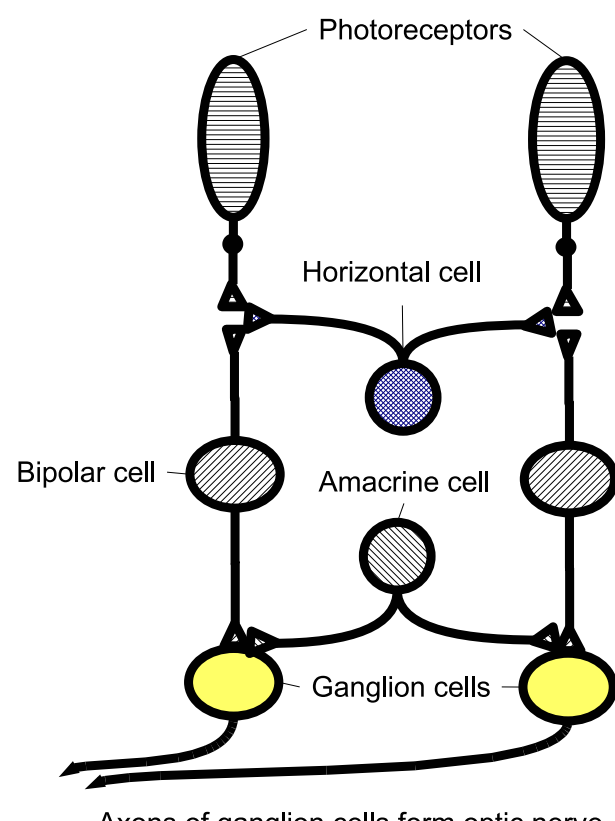

Fig. 1. Simplified cross section of a retina showing horizontal inhibitory cells

\section{BACKGROUND}

A. What is known about biological lateral inhibition and adaption of the mammalian eye?

The structure of biological retina has been clearly described (6) with light sensitive rod and cone cells being connected to bipolar cells. These then connect to ganglion cells which form part of the optic nerve, see Fig. 1. Lateral inhibitory cells, called amacrine cells, have also been identified; these have an important role in edge detection (7) and it has been shown that lateral inhibition is important in allowing the retina to operate over a wide range of light intensities (8).

Lateral inhibition enhances the effects of point light sources by inhibiting the output of neighboring neurons.

Other mechanisms which also allow the mammalian eye to adapt to wide ranges of light intensity include chemical changes in the neurons of the retina and physical changes in 


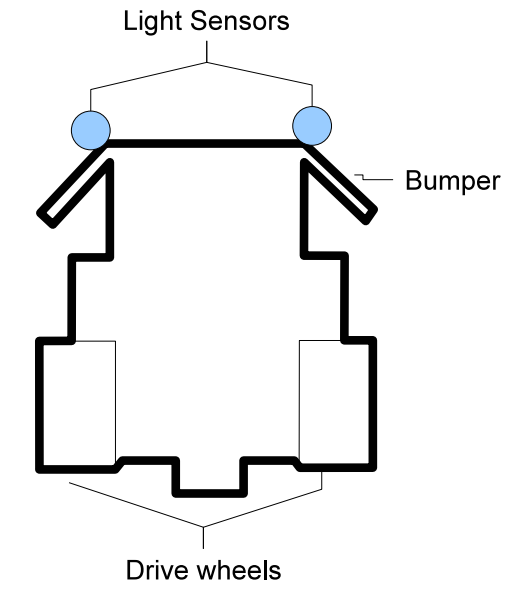

(a) Schematic of the robot

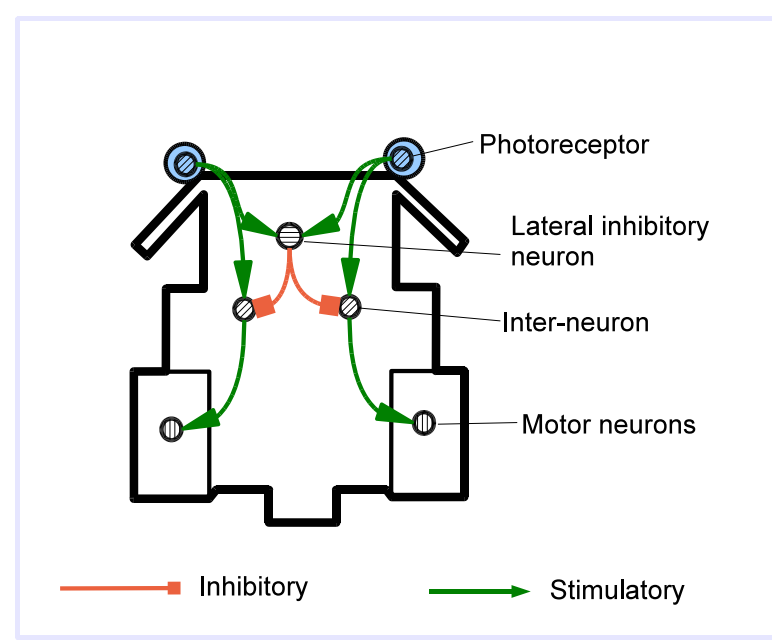

(b) Layout of neurons

Fig. 2. A schematic of the robot. (a) indicates the position of the two light sensors and motors (b) shows the location of the photoreceptors, lateral inhibitory neuron and the two inter-neurons which attach to the motors. Excitatory connections are indicated by an arrow and inhibitory connections are indicated by a block. Both light sensors excite the single inhibitory neuron which is attached to the two excitatory inter-neurons. Note the interneurons are excitatory which causes the vehicle to perform negative phototaxis.

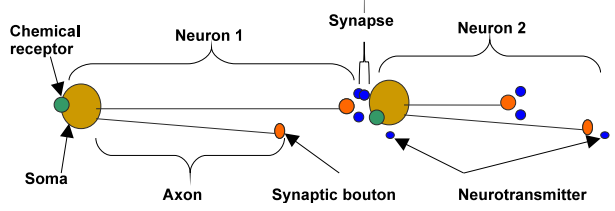

Fig. 3. Diagram showing two of our simplified spiking neurons the shape/structure of the eye (iris closure) (6). Collectively the mechanisms provide the human eye with a contrast ratio of 1,000,000:1 (6).

\section{B. Sensor normalization techniques}

Sensor normalization is a standard precursor to practical robot experiments. It involves pre-determining the maximum and minimum values that a sensor will detect in the robots environment. These are then used to produce a standard range of sensor outputs, typically 0.0 representing the minimum normalised reading to 1.0 representing the maximum normalised sensor reading (9).

If $\min =$ minimum sensor value, $\max =$ maximum sensor value, $i=$ current sensor reading and $i_{n}=$ normalised sensor reading then :

$$
i_{n}=\frac{i-\min }{\max -\min }
$$

This strategy of predetermining the range of sensor readings works well when the environment is known and unchanging, but is less successful when either the environment is unknown or the minimum and maximum sensor values vary over time, such as light levels during a day. Mammalian eyes successfully adapt to varying light ranges throughout the day and it is hoped that using lateral inhibition may improve the range of conditions in which a robot may function effectively without the need to predetermine the minimum and maximum sensor readings.

Previous experiments (4) with simulated negatively phototaxic robots have had limited success due to over-excitation of neurons by the sensors' values. It is hoped that using lateral inhibition will allow the robots to consistently perform orientation tasks across a wider range of light intensities.

\section{Our event driven integrate and fire model}

Our model uses a simple neuron with many axons and no dendrites. In biological neurons, the soma would have only one axon which would subsequently branch, this is functionally the same as having a soma with many (one or more) axons as in Fig. 3 shows 2 neurons each with two axons.

Simulated spiking neurons typically model the membrane at many points on the neuron in small time steps. This allows for very localised effects to be reproduced. For a non-branching axon, the nature of the spike progression is largely deterministic i.e. once a spike has started in a region, an impulse will propagate along the axon to the synaptic boutons. The amount of time between a spike being initiated in the postsynaptic membrane and neurotransmitter being released by the same neuron from its presynaptic membrane should be related to only the length of the axons for a particular neuron. Once a spike has been initiated, it is possible to determine the point in time when the synaptic boutons should release neurotransmitter; this is designated as a neurotransmitter release event. The time-consuming calculations associated with modelling many points on the neuron membrane can therefore be restricted to modelling 
the postsynaptic membrane of each synapse and subsequent simulation of neurotransmitter release at the presynaptic boutons.

The action of the postsynaptic membrane can be further simplified by assuming that the refractory period of the membrane is fairly constant and that a spike is produced if the internal potential rises above a given threshold. Hence, when a neurotransmitter is detected by the receptors, a set amount of inward movement of ions is produced which will change the internal potential by a known amount which may or may not be above threshold. If it is above threshold, an impulse is simulated which by the above argument will cause a neurotransmitter release event to occur in the future. If the postsynaptic membrane has spiked, another spike will not occur for a period of time known as the refractory period. Excitation of the postsynaptic receptors will not cause a new spike to occur, but may change the potential across the membrane. At the end of the refractory period, a new spike may occur if the potential across the postsynaptic membrane is above threshold.

The above simplifications result in an event-driven model where processing only occurs when an event is due which is similar to work by Rochel (10). The main two events which need to be modelled for a neuron are :

1) The release of neurotransmitter by the presynaptic membrane; which immediately causes receptors on a connecting postsynaptic neuron to be activated and ion transport through the membrane which may result in the initiation of a spike.

2) The end of a refractory period for a postsynaptic membrane; which may result in a new spike and refractory period.

When either event is raised, the potential inside the soma must be recalculated, this is because time will have passed and the potential will have decayed.

\section{RESEARCH QUESTIONS}

These are the research questions identified:

\section{A. Does a lateral inhibitory neuron produce response across a wider range of light intensities?}

This question is motivated by previous experiments that had not achieved signicant rotation towards dark areas, in a negative phototaxic robot (4)

B. Do we need a membrane reset when a spike fires with lateral inhibition?

Integrate and fire neurons typically reset the membrane voltage to a preset resting potential. In both (5) and (4) the neurons were not reset. We therefore applied the same strategy along with the standard reset model to compare performance.

C. Can a simple linear decay model for leakiness produce rotation?

The aim of our initial studies (5) and (4) had been to identify a simple spiking model with the simplest calculations which will produce appropriate behaviour for phototaxis. It is hypothesized that lateral inhibition will reduce the oversaturation of neurons which use a linear model of voltage leakiness across the neuron membrane.

\section{Methodology}

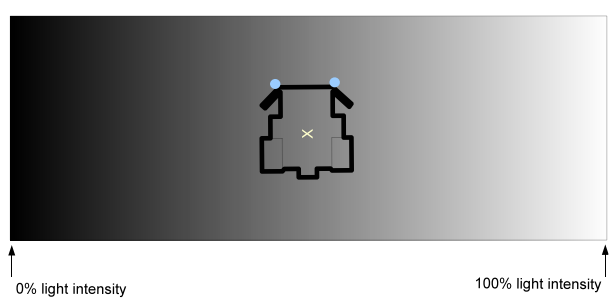

Fig. 4. The robot on the linear light gradient. The robot is placed orthogonally to the gradient and is 'pinned' in position so that it can only rotate around the centre (cross on the diagram).

The experiment to test the effect of using a lateral inhibitory neuron was carried out on a simulated robot. A schematic of the robot is shown in Fig. 2(b).

Simulated receptor neurons were attached to light sensors. These sensory neurons then connected to inter-neurons which subsequently attached to the motors with excitatory ipsilateral connections (left receptor connects to left motor). The sensory neurons both fed into an additional inhibitory neuron attached to the inter neurons which reduced their output. At the start of each experiment the robot was placed perpendicular to a linear light gradient, see Fig. 4.

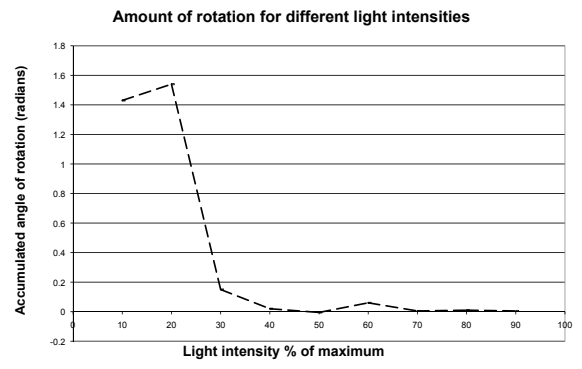

Fig. 5. A graph of the amount of rotation achieved in the previous experiment without any lateral inhibition. It can be clearly seen that once the light intensity reaches $20 \%$ of maximum, any subsequent increase in light intensity results in rapidly falling rotation. In particular, once the light intensity is $40 \%$ of maximum, the robot fails to rotate. The reason for this is that both photoreceptors are saturated. Note that the robot is never placed right at the edge of the environment as any rotation could then cause a physically impossible configuration.

The simulation prevented forward motion of the robot, but allowed rotation so that the robot would spin on the spot (for example an insect being tested for pheromone orientation in a wind tunnel). The simulation was run for 10 seconds and the total angle of rotation was recorded. The robot was placed at incremental distances from the left side of the gradient, providing 99 data samples. Each experiment was repeated 10 times, resulting in a total of 990 results.

The experiment was first carried out with neurons which reset the membrane voltage and then with neurons which did not reset the membrane voltage. 


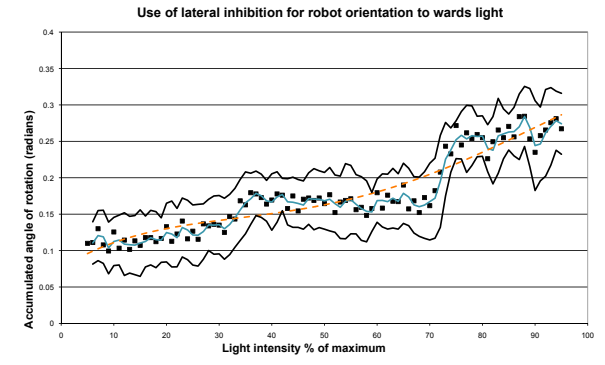

(a) Neuron with a membrane reset function

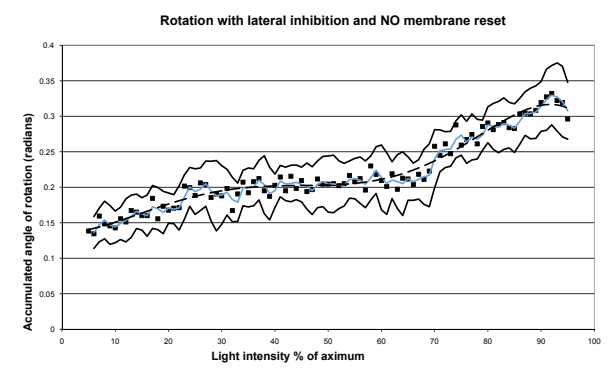

(b) Neuron without a membrane reset function

Fig. 6. Both of these graphs illustrate performance for the neural network that contains a lateral inhibitory neuron. The top diagram is for the model that does include a reset for the membrane potential, the bottom diagram does not included a reset for the membrane potential. The graphs showing the amount of rotation achieved for different light intensities showing a small and consistent amounts of rotation across the entire range of light intensities. Each graph plots the average results for each light intensity with a feint line showing a rolling average which is bounded by two solid dark lines showing the rolling average for one standard deviation. A dotted polynomial trendline has also been fitted to the average values.

\section{REsults}

Our earlier result in which the model has no lateral inhibition is shown in Fig. 5 This shows the amount of rotation achieved when the light intensity is increased. It can be clearly seen that once the light intensity reaches $20 \%$ of maximum, any subsequent increase in light intensity results in rapidly falling rotation. In particular, once the light intensity is $40 \%$ of maximum, the robot fails to rotate. The reason for this is that both photoreceptors are saturated. Note that the robot is never placed right at the edge of the environment as any rotation could then cause a physically impossible configuration. This is believed to be caused by the neurons saturating. The new results with lateral inhibition are given in Fig. 6. Each graph plots the average results for each light intensity with a feint line showing a rolling average which is bounded by two solid dark lines showing the rolling average for one standard deviation. A dotted polynomial trendline has also been fitted to the average values. Fig. 6(a) is the first experiment where a membrane reset function has been used and Fig. 6(b) gives the results for the second experiment where the membrane voltage is not membrane reset after a spike is initiated in the neuron.

These show that :

1) Rotation occurs across all light intensities compared to the lower $20 \%$ of the light intensities in previous results shown in Fig. 5 .

2) The accumulated angle of rotation is fairly uniform across all light intensities.

3) The variance of the results is small and uniform across the entire range of intensities.

4) The absence of a reset function produces greater accumulated angles of rotation.

\section{DISCUSSION}

\section{A. Does an inhibitory neuron produce rotation across a wider range of light intensities?}

Our results show that lateral inhibition produces rotation across all light intensities. This can be achieved with our simplified spiking neuron by the addition of one extra inhibitory neuron. The magnitude of rotation is reduced, but the consistency of rotation for all light intensities allows scaling of the motor outputs to produce appropriate levels of rotation.

\section{B. Other advantages of lateral inhibition.}

1) Do we need a membrane reset when a spike fires with lateral inhibition?

The graphs show that a membrane reset function lowered the accumulated angle of rotation, this is due to the absence of repeat firings that were found in the "no membrane reset" experiments. This would suggest that a membrane reset function is not essential for negative photo-taxis. An additional observation is that the graph for no membrane reset was smoother.

2) Can a simple linear decay model for leakiness produce rotation?

In previous experiments, neurons with exponential voltage leakiness tended to saturate less quickly than neurons with linear decay and therefore resulted in rotation over a wider range of light intensities. In the experiments with lateral inhibition, the simulated robot rotated across the entire range of light intensities. Lateral inhibition has had the added benefit of preventing neurons from saturating thus eliminating the need for a more computationally expensive self-regulating exponential voltage decay function.

\section{Summary}

Taken over all, the lateral inhibition has been shown to be useful, in particular:

1) Lateral inhibition allowed the robot to operate across the entire range of light intensities.

2) Lateral inhibition removed the need for a membrane reset function in the simplified integrate and fire neuron

3) Lateral inhibition removed the need for computationally expensive exponential decay function.

The neuron can be simplified to having a linear voltage decay model with no membrane reset function when the neural 
network includes lateral inhibition. This is the computationally simplest integrate and fire spiking model that we have produced which produces phototaxic responses over a wide range of light intensities.

\section{FUTURE WORK}

The findings from this study will now be used in a robot experiment in an environment which contains a light gradient representing food. The neural network will increase in complexity to allow for positive phototaxis when the robot is 'searching for food'.

Once this has been achieved, we aim to investigate using a regulatory genetic network which will evolve similar neural networks to the one used in this and the subsequent experiments using techniques developed by Wróbel (11).

\section{REFERENCES}

[1] R. French and L. Cañamero, "Introducing neuromodulation to a braitenberg vehicle," Robotics and Automation, 2005. ICRA 2005. Proceedings of the 2005 IEEE International Conference on, pp. 4188-4193, April 2005.

[2] L. Cañamero, O. Avila-Garcia, and E. Hafner, "First experiments relating behavior selection architectures to environmental complexity," Intelligent Robots and Systems, 2002. IEEE/RSJ International Conference on, vol. 3, pp. 3024-3029 vol.3, 2002.

[3] Bisset, "The dynamics of photo-taxis: Applying the agent envi-ronment interaction system to a simple braitenberg robot," in European Conference on Artificial Life, vol. 4, 1997, pp. 327-336.

[4] D. Bowes, R. Adams, V. Steuber, L. Cañamero, and N. Davey, "Analysis of soma leakiness and receptor response to stimulus in a simulated spiking neuron," in ANNIP. INSTICC, 2008, pp. 100-106.

[5] D. Bowes, R. Adams, V. Steuber, L. Canamero, and N. Davey, "A simplified spiking neural network for a robot." Science and Technology Research Institute, University of Hertfordshire., Tech. Rep., 2007.

[6] J. T. Kandel ER, Schwartz JH, Principles of Neural Science, 4, Ed. McGraw-Hill, New York, 2000.

[7] A. L. Jacobs, Frank, S. Werblin, and A. L, "Principles of communications," 1965.

[8] M. V. Srinivasan, S. B. Laughlin, and A. Dubs, "Predictive coding: A fresh view of inhibition in the retina," Proceedings of the Royal Society of London. Series B, Biological Sciences, vol. 216, no. 1205, pp. 427-459, 1982. [Online]. Available: http://www.jstor.org/stable/35861

[9] B. Bagnall, Maximum Lego NXT. Variant Press, 2007.

[10] O. Rochel and D. Martinez, "An event-driven framework for the simulation of networks of spiking neurons," in European Symposium in Artificial Neural Networks, 2003, pp. 295-300.

[11] M. Joachimczak and B. Wróbel, "Evo-devo in silico: a model of a gene network regulating multicellular development in 3d space with artificial physics," in Artificial Life XI: Proceedings of the Eleventh International Conference on the Simulation and Synthesis of Living Systems, S. Bullock, J. Noble, R. Watson, and M. A. Bedau, Eds. MIT Press, Cambridge, MA, 2008, pp. 297-304. 